Research Article

\title{
Time-Frequency Analysis and Application of a Vibration Signal of Tunnel Excavation Blasting Based on CEEMD-MPE-HT
}

\author{
Chen-yang Ma $\mathbb{D}^{1},{ }^{1}$ Li Wu $\mathbb{D},{ }^{1}$ Miao Sun $\mathbb{D}^{1},{ }^{1}$ and Qing Yuan $\mathbb{D}^{2}$ \\ ${ }^{1}$ Engineering Research Center of Rock-Soil Drilling \& Excavation and Protection of the Ministry of Education, \\ Faculty of Engineering, China University of Geosciences, Wuhan 430074, Hubei, China \\ ${ }^{2}$ CCCC Second Harbour Engineering Co., Ltd., National Enterprise Technology Center, Wuhan, China
}

Correspondence should be addressed to Li Wu; lwu@cug.edu.cn

Received 21 December 2020; Revised 11 January 2021; Accepted 25 January 2021; Published 4 February 2021

Academic Editor: Qi ZHAO

Copyright ( $\odot 2021$ Chen-yang Ma et al. This is an open access article distributed under the Creative Commons Attribution License, which permits unrestricted use, distribution, and reproduction in any medium, provided the original work is properly cited.

\begin{abstract}
The traditional empirical mode decomposition method cannot accurately extract the time-frequency characteristic parameters contained in the noisy seismic monitoring signals. In this paper, the time-frequency analysis model of CEEMD-MPE-HT is established by introducing the multiscale permutation entropy (MPE), combining with the optimized empirical mode decomposition (CEEMD) and Hilbert transform (HT). The accuracy of the model is verified by the simulation signal mixed with noise. Based on the project of Loushan two-to-four in situ expansion tunnel, a CEEMD-MPE-HT model is used to extract and analyze the time-frequency characteristic parameters of blasting seismic signals. The results show that the energy of the seismic wave signal is mainly concentrated in the frequency band above $100 \mathrm{~Hz}$, while the natural vibration frequency of the adjacent existing tunnel is far less than this frequency band, and the excavation blasting of the tunnel will not cause the resonance of the adjacent existing tunnel.
\end{abstract}

\section{Introduction}

As is known to all, the physical quantities such as peak velocity of particle vibration (PPV), acceleration (a), and displacement $(s)$ are usually used to measure and determine the degree of influence of structures under blasting vibration [1]. However, the safety standard of blasting vibration of existing regulations is relatively broad and empirical. Therefore, many scholars have proposed and improved safety control criteria for blasting engineering from the perspectives of numerical calculation [2], stress wave propagation theory [3], and maximum instantaneous input energy angle of structure [4]. In engineering practices, in addition to the blasting peak vibration velocity as the basis for controlling blasting hazards, the influencing factors of the vibration degradation of the protected construction (building) on the duration of blasting vibration, frequency of blasting vibration, the energy released by blasting vibration, and type of adjacent structures cannot be ignored [5].
However, blasting seismic waves is a typical nonstationary and nonlinear signal, and only with the help of the time-frequency analysis method can the monitored timedomain signals be transformed into frequency-domain signals. Further interpretation of signal frequency-domain information and energy distribution characteristics is of great significance for blasting seismic wave hazard control. Common time-frequency analysis methods include the short-time Fourier transformation, Wigner-Ville distribution, continuous wavelet transform, etc. $[6,7]$. The theoretical basis of the abovementioned methods is Fourier transform, which inevitably leads to false frequencies and redundant signal components $[8,9]$. In view of this problem, Huang et al. [10] proposed empirical mode decomposition (EMD) in 1998, which fundamentally broke through the limitation of Fourier transform theory and established a signal analysis method based on instantaneous frequency for the first time $[11,12]$. The process of decomposition retained the characteristics of the data itself. 
But, there are some problems with EMD. Considering that the monitoring signals of blasting seismic waves are mostly noisy signals, the mixing of noise makes the EMD results produce modal confusion [13], which makes it difficult to identify the time-frequency characteristics of measured blasting seismic wave signals. To solve the abovementioned problems, the efficient random signal detection method of multiscale permutation entropy (MPE) is introduced in this paper. Combined with the optimized EMD, complementary ensemble empirical mode decomposition (CEEMD), the joint algorithm of CEEMD-MPE is obtained. The time-frequency analysis model of CEEMDMPE-HT can be established by applying the Hilbert transform (HT) to IMF obtained by the CEEMD-MPE joint algorithm. This model can realize the time-frequency analysis of the noise signal and extract the real energy and frequency information of the signal.

This paper discusses the application of the CEEMDMPE-HT time-frequency analysis model in the blasting project of the Loushan tunnel, which is a separated tunnel with a large span and small clear distance. Compared with other time-frequency analysis methods, the proposed timefrequency algorithm not only preserves the real information of the signal but also has the advantage of effectively suppressing the mode confusion. The advantages of this timefrequency analysis model are compared and verified in the mixed simulation signals and successfully applied in practical engineering. According to the time-frequency characteristic parameters obtained by the proposed model algorithm, the influence of expanding blasting on one side of a super-large and small clear distance separated tunnel on the other side is discussed. The time-frequency algorithm provides a useful idea for solving the identification of blasting seismic wave signals, the analysis of time-frequency characteristic parameter law, and the control of blasting vibration hazards in engineering practices.

\section{CEEMD-MPE-HT Time-Frequency Analysis Model}

2.1. The Principle of CEEMD. CEEMD [14] is the method of adding two white noise signals $n(t)$ in opposite directions to the original monitoring signal $S(t)$. Generally, 0.2 times of the standard deviation of $S(t)$ is taken as the white noise signals, and empirical mode decomposition (EMD) [10] is performed, respectively. follows:

The specific implementation steps of CEEMD are as

Step 1: white noises are added in opposite directions to $S(t)$ in pairs to get two new signal sources, $S(t)+n(t)$ and $S(t)-n(t)$, respectively

Step 2: EMD decomposition of $S(t)+n(t)$ and $S(t)-$ $n(t)$

Step 3: step 1 and step 2 are repeated until the set number of adding white noise is reached
Step 4: the overall average of all the results obtained in Step 2 is carried out, and the obtained result is the CEEMD result

Since white noise in opposite directions is added in pairs and the IMF is averaged many times, it can be considered that the artificially introduced white noise has been eliminated. This method is an improvement of ensemble empirical mode decomposition (EEMD), which not only ensures that the decomposition effect is equivalent to EEMD [15] but also reduces the reconstruction error caused by white noise at the same time.

2.2. The Principle of MPE. Permutation entropy (PE) [16] is a method to detect randomness and dynamic mutation in time series. It has the advantages of a simple concept, fast calculation speed, and strong anti-interference ability, especially suitable for the analysis of nonlinear data.

MPE [17] is an optimization method of combining multiple scales with PE. It can analyze the time series more efficiently.

Suppose the length of the any one-dimensional time series $x(i)$ is $L . X(i)$ can be expressed as $x_{L}(i)=\left\{x_{1}, x_{2}\right.$, $\left.x_{3}, \cdots x_{L}\right\}$. Equation (1) is obtained by coarsening $x_{L}(i)$ with a multiscale:

$$
y_{j}^{s}=\frac{1}{s} \sum_{i=(j-1) \cdot s+1}^{j \cdot s} x_{i}, \quad 1 \leq j \leq \frac{L}{s} .
$$

In equation (1), $s$ is the scale factor and $y_{j}^{s}$ is the multiscale time series. When $s=1, x(i)$ does not change. The core of multiscale coarse granulation is to segment the time series and take the average value of each segment to improve the operation accuracy. So, the value of $s$ is critical. When $s$ is too large, it cannot reflect the complexity of the time series. When $s$ is too small, it is difficult to extract fragments and the analysis effect is poor. According to [18], this paper takes $s=10$. The MPE value $[19,20]$ of $x(i)$ can be obtained by calculating the PE value of $y_{j}^{s}$ in equation (1).

According to the definition of MPE and [21, 22], when the MPE of $x(i)$ is greater than 0.6 , it can be considered as an abnormal component with great randomness and needs to be eliminated.

2.3. The Principle of HT. The detailed process of HT is introduced in [10]. The Hilbert marginal spectrum is mainly discussed in this paper, which is obtained by integrating the Hilbert time spectrum with respect to time, as shown in equation (2). The marginal spectrum is the global amplitude of each frequency, which represents the total cumulative amplitude in the statistical sense. $H(w, t)$ is the spectrum of Hilbert time [23], where $w$ denotes frequency.

$$
h(\omega)=\int_{0}^{T} H(\omega, t) \mathrm{d} t
$$




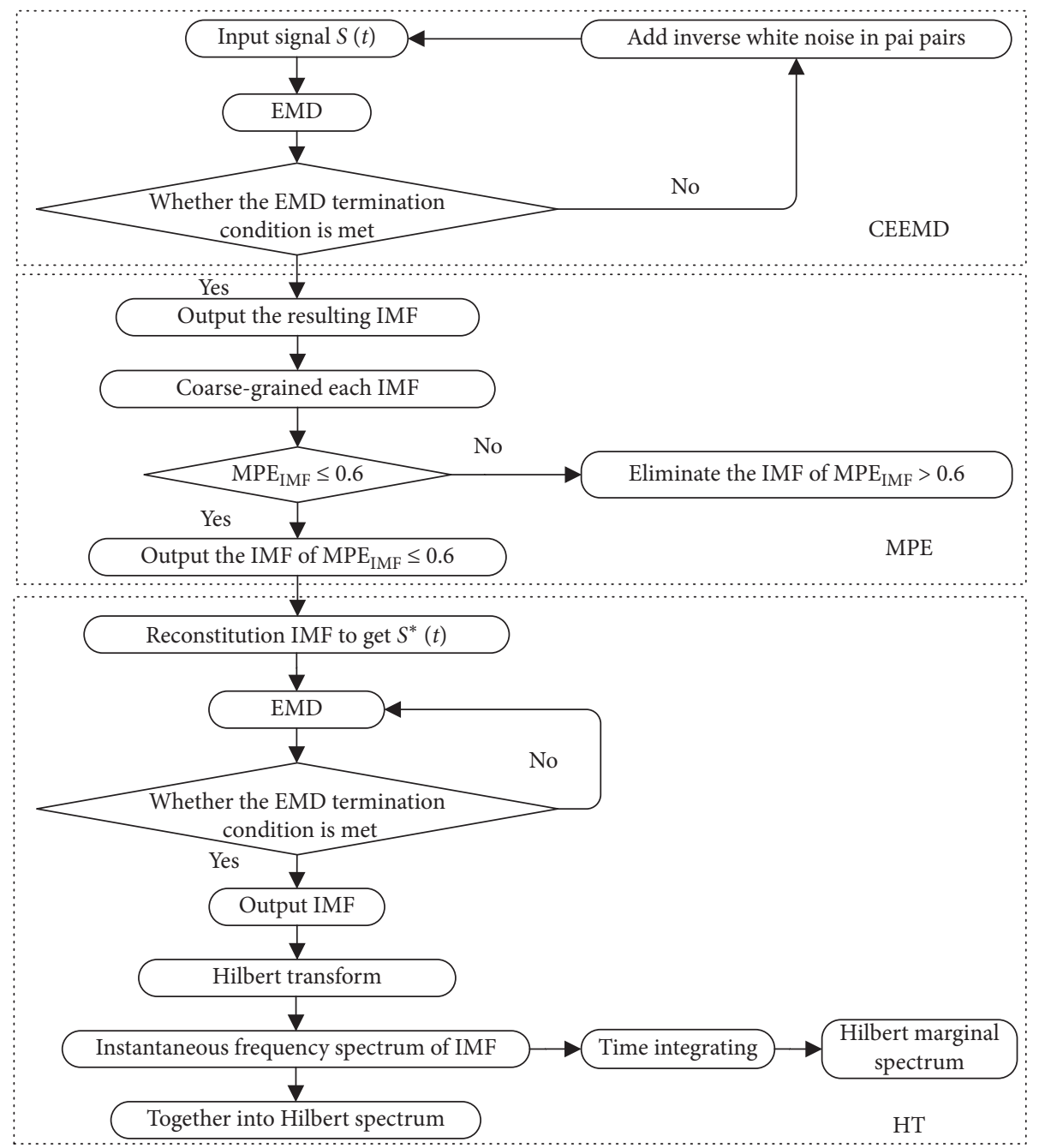

FIgure 1: Flow chart of the CEEMD-MPE-HT time-frequency analysis model.

Therefore, the flow chart of the CEEMD-MPE-HT timefrequency analysis model of seismic wave vibration signal can be established (Figure 1). For the termination conditions of EMD, refer [10].

\section{Time Frequency Analysis of a Simulation Signal with Noise}

3.1. The Establishment of a Simulation Signal with Noise. The noise simulation signal is composed of Gaussian white noise with a power of 0.2 and sinusoidal steady-state signal with a frequency of 120 . The number of samples $N=1028$, and $t$ is a time series with a total length of $1 \mathrm{~s}$ and a unit time interval of $1 / N$. Figure 2 shows the waveform of the simulation signal.

3.2. Comparison of the Time-Frequency Analysis Results of the Simulation Signal with Noise. Three time-frequency analysis methods were used to analyze the simulation signal shown in Figure 2. The results can be seen in Figures 3-5, among which Figures 3(b), 4(b), and 5(b) are the results of the modal decomposition of the IMF marginal spectrum. The $Y$ axis coordinate is $E S D$ represents the energy spectral density.

By observing Figures 3-5, the following conclusions can be drawn:

(1) There is an obviously one-to-one correspondence between the results of modal decomposition and the IMF marginal spectrum.

(2) The IMF high-frequency modes obtained by EMD are seriously confused. The addition of white noise results in the appearance of false IMF components in the decomposition, such as the IMF1 and IMF2 components with frequencies greater than $120 \mathrm{~Hz}$. The low-frequency decomposition is relatively stable.

(3) The high-frequency modal confusion of IMF obtained by CEEMD is somewhat reduced, but there is a slight modal split at low frequencies [24], such as IMF6 and IMF7.

(4) The phenomenon of IMF modal confusions obtained by CEEMD-MPE was inhibited. The components are arranged from high frequency to low frequency, and the decomposition results are good. From the 


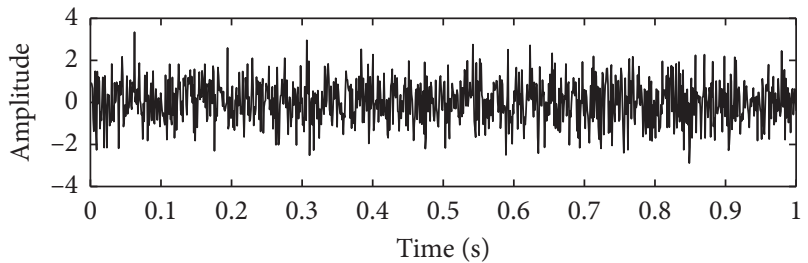

(a)

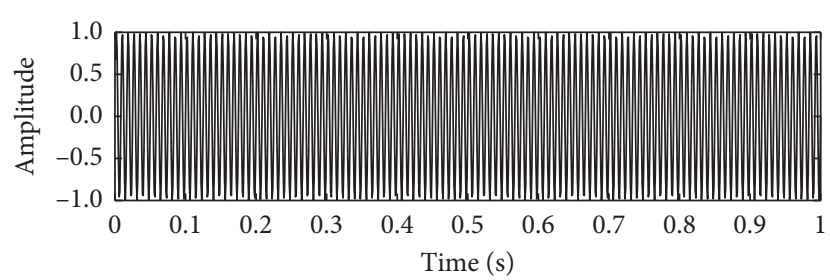

(b)

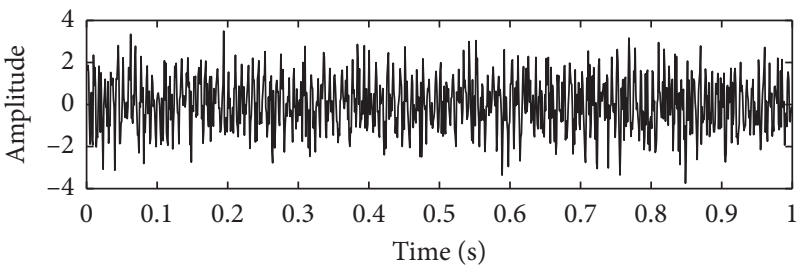

(c)

Figure 2: Waveform of the simulation signal. (a) Gaussian white noise with power of 0.2. (b) Sinusoidal steady-state signal with frequency $120 \mathrm{~Hz}$. (c) The simulation signal.
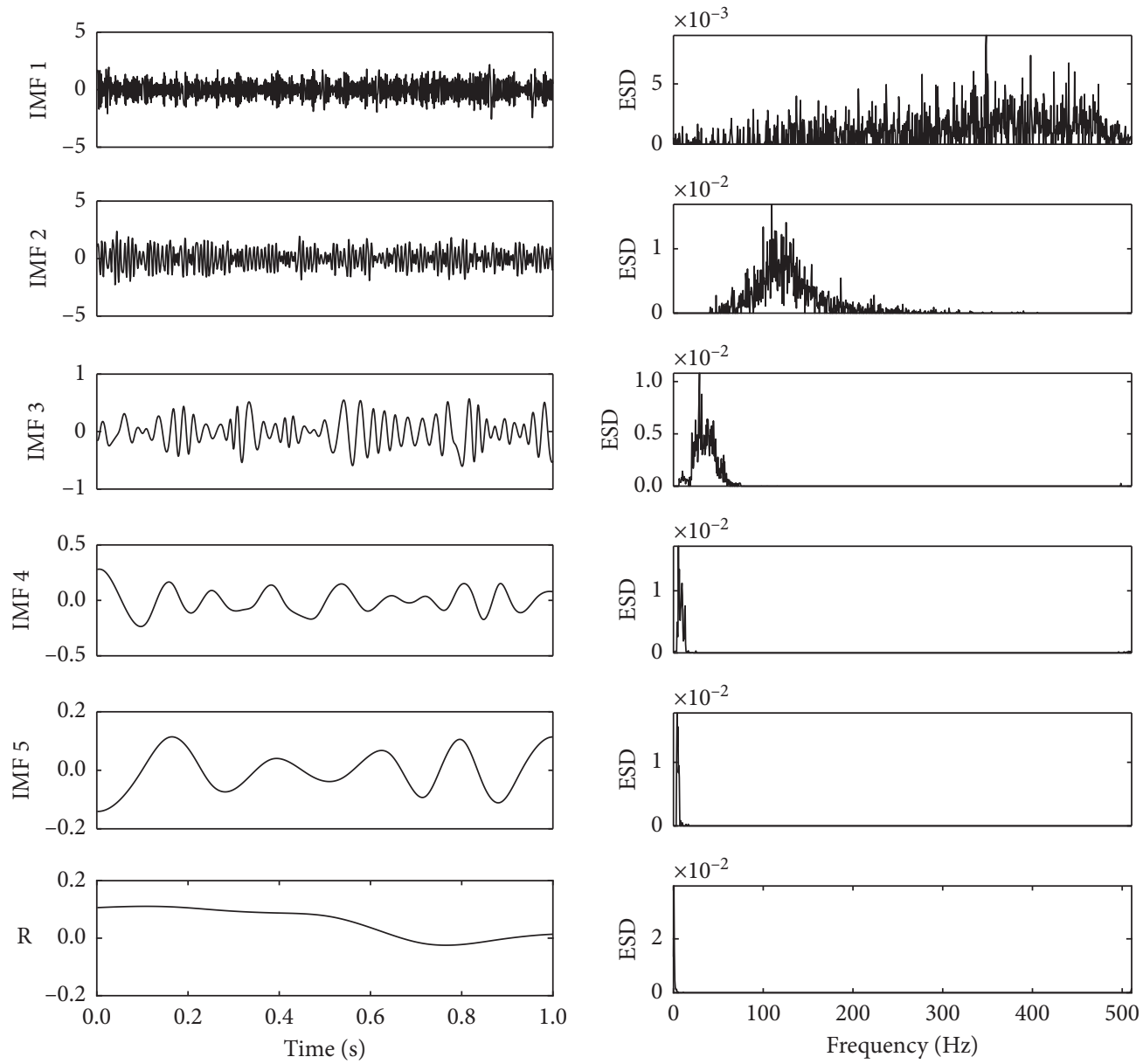

(a)

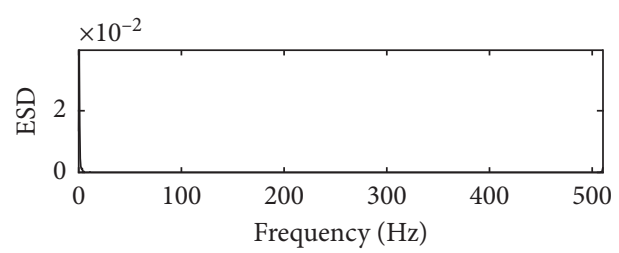

(b)

FIgURE 3: Results of EMD-HT time-frequency analysis. (a) Decomposition results of EMD. (b) IMF marginal spectrum. 

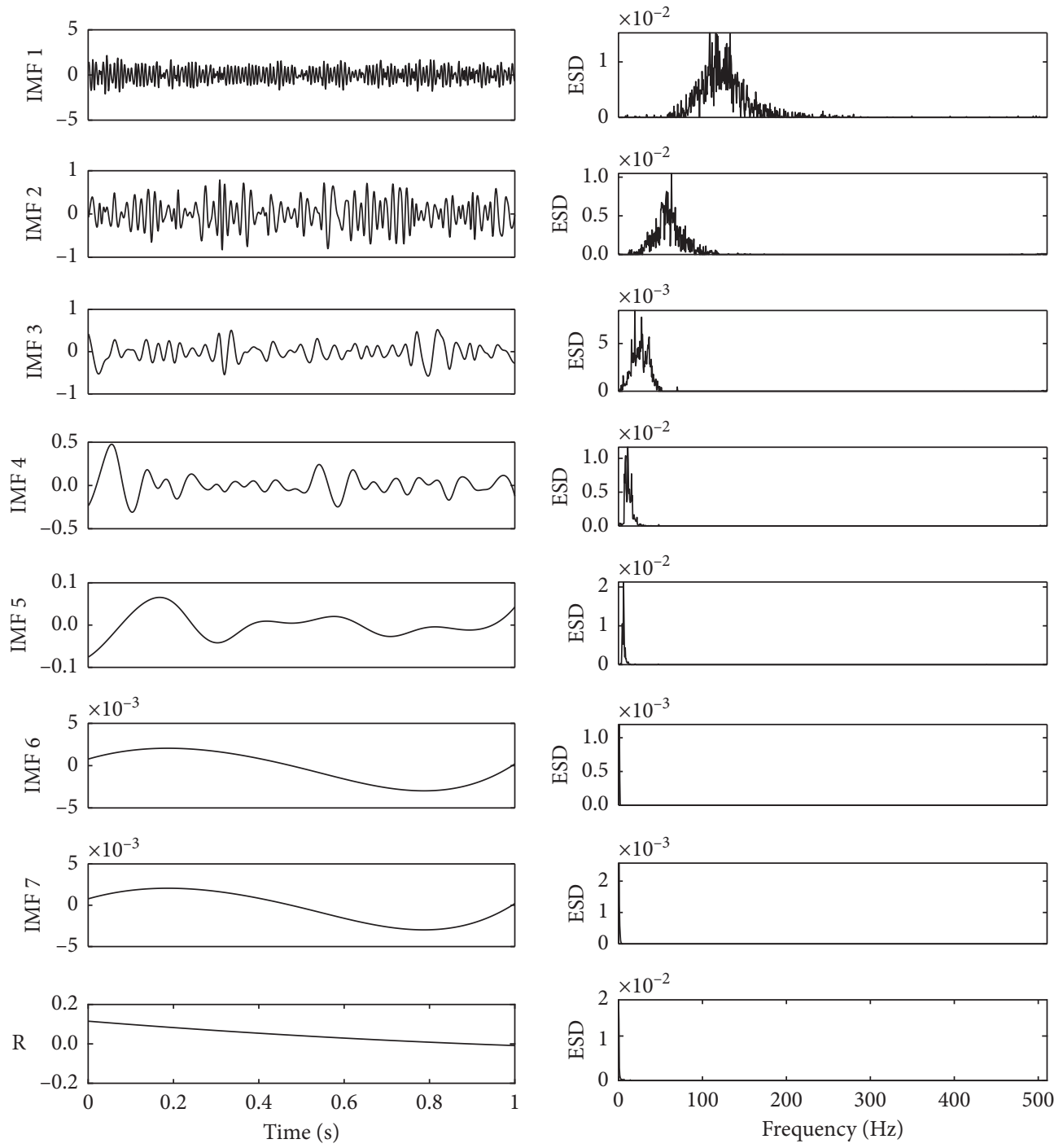

(a)

(b)

Figure 4: Results of CEEMD-HT time-frequency analysis. (a) Decomposition results of EMD. (b) IMF marginal spectrum.

marginal spectrum, it can be clearly concluded that the $120 \mathrm{~Hz}$ sinusoidal steady-state signal energy of the original signal is mainly contained in IMF1 IMF4.

The processed results of the simulation signal with noise obtained by the abovementioned time-frequency analysis models are compared. It can be found that the IMF mode confusion obtained by EMD is serious; compared with EMD, the mode confusion of CEEMD in high frequency has been greatly improved, but there is still a mode splitting phenomenon in low frequency; the IMF obtained by CEEMD-MPE has good stability in both high frequency and low frequency. At the same time, it can be found that the CEEMD-MPE-HT model can reflect the real energy and frequency information of the signal and is more suitable for time-frequency analysis of noisy signals.

\section{Engineering Application}

4.1. Engineering Application. The Loushan tunnel is part of the first construction section of the highway project from Jiaojiang to the east of Wenling City. It is a separate tunnel. In order to meet the traffic demand, the original two-way four-lane tunnel was expanded into a two-way eight-lane tunnel in situ. During the closed construction of the right tunnel, the left tunnel shall remain open to traffic normally. Relevant design parameters are shown in Table 1. Therefore, dynamic monitoring must be carried out for the existing left 

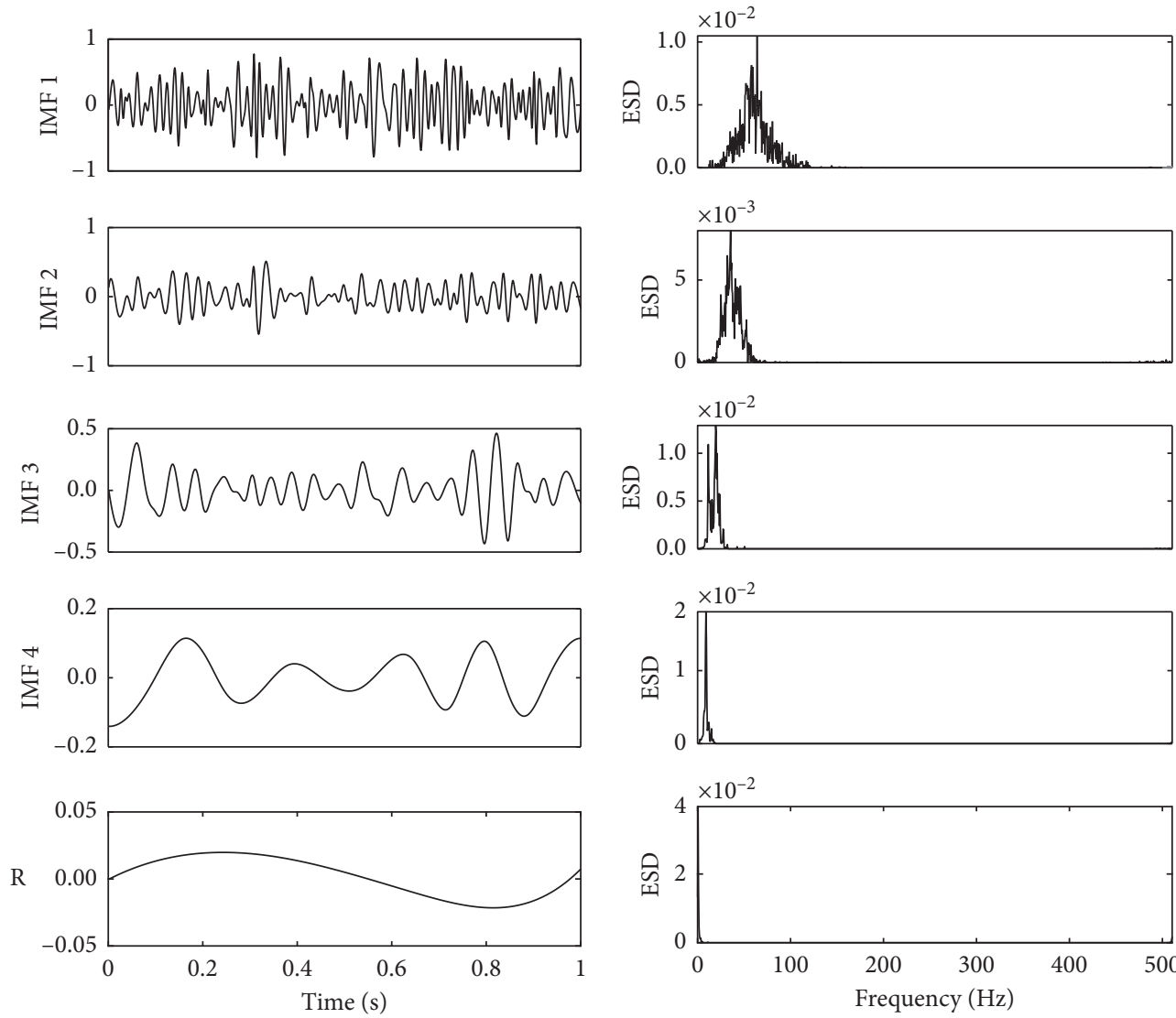

(a)

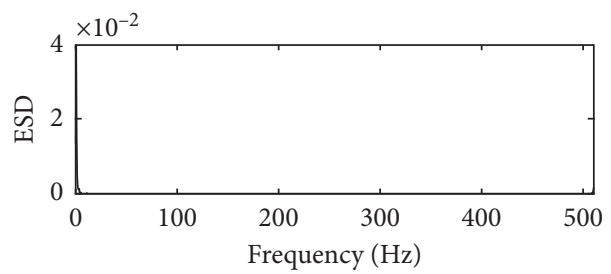

(b)

Figure 5: Results of CEEMD-MPE-HT time-frequency analysis. (a) Decomposition results of EMD. (b) IMF marginal spectrum.

TABle 1: Basic design parameters of expanded excavation of the Loushan tunnel (unit: $\mathrm{m}$ ).

\begin{tabular}{lcc}
\hline Project & Loushan tunnel after expansion & Loushan tunnel before expansion \\
\hline Section form & Two-way eight-lane & Two-way four-lane \\
Width of carriageway $(\mathrm{m})$ & $2 \times 3.50+2 \times 3.75$ & $2 \times 3.75$ \\
Lateral dimension $(\mathrm{m})$ & 0.50 & 0.50 \\
Width of maintaining roadway $(\mathrm{m})$ & 0.75 & 0.75 \\
Total width $(\mathrm{m})$ & 17.25 & 10.25 \\
Tunnel clearance $(\mathrm{m})$ & 5.00 & 5.00 \\
\hline
\end{tabular}

tunnels to ensure normal traffic during the right tunnel expansion blasting construction. Besides, the influence of the right tunnel expansion blasting on the left tunnel must be strictly controlled.

4.2. Blasting Monitoring Program. The purpose of blasting vibration monitoring is to ascertain the impact of blasting vibration on surrounding structures, timely feedback of the dynamic monitoring information, and to provide a reliable basis for improving the blasting design, controlling the blasting scale, and guiding the blasting construction. The Ubox-5016 intelligent blasting vibration monitor is adopted for blasting vibration monitoring, and the layout of measuring points is shown in Figure 6.
4.3. Analysis of the Measured Blasting Vibration Signal Based on CEEMD-MPE-HT. In order to analyze the time-frequency characteristics and energy characteristics of blasting seismic wave signals in tunnel expansion and excavation, a typical blasting vibration signal is selected as the analysis object in combination with the on-site blasting vibration monitoring scheme, as shown in Figure 7.

Time-frequency analysis based on CEEMD-MPE-HT was performed for radial seismic wave signals in Figure 7(a), and the result is shown in Figure 8. From Figure 8(a), it can be seen that the IMFs decomposed by radial seismic wave signal CEEMD-MPE rank from high to low frequencies. Each IMF component is relatively stable, and the result is consistent with the simulation signal CEEMD-MPE decomposition result. Combined with the IMF marginal 


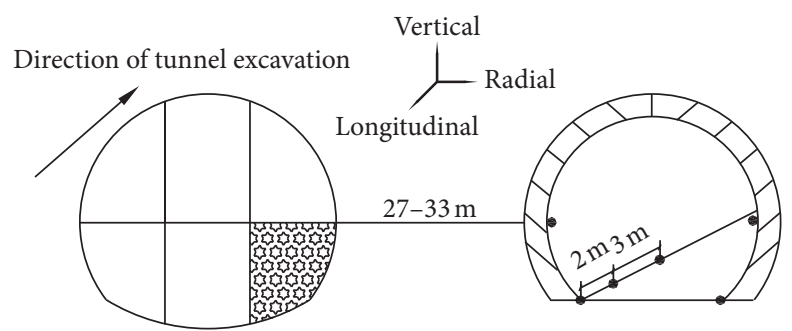

The right line of Loushan tunnel

The left line of Loushan tunnel

- Measuring point

[ing Explosive source

$\mathbb{8}$ The lining

FIgURE 6: Layout of measuring points.

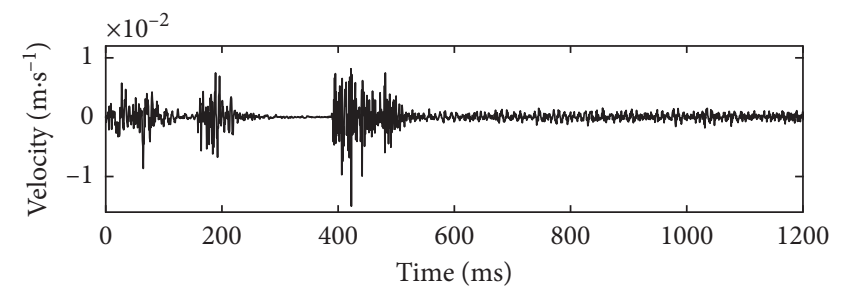

(a)

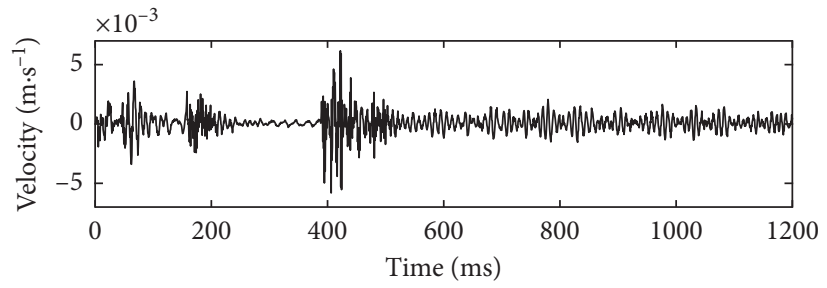

(b)

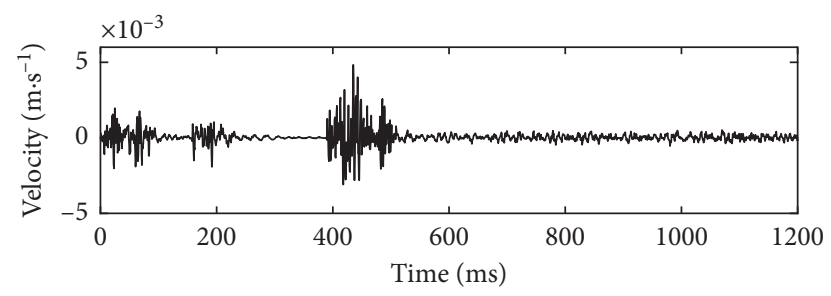

(c)

FiguRE 7: Measured typical seismic wave signal map. (a) Radial seismic wave signals. (b) Longitudinal seismic wave signals. (c) Verticle seismic wave signals.

spectrum of the radial seismic wave signal in Figure 8(b), it can be seen that the IMF1 and IMF2 are high-frequency components distributed in the frequency range above $100 \mathrm{~Hz}$ and carry the main energy of the radial seismic wave signal. IMF3 IMF5 are middle- and low-frequency components, and the energy contained in them is relatively small compared with IMF1 and IMF2. $R$ is both the residual term and the trend term. In Hilbert transformation, the residual component is generally not considered.

According to the marginal spectrum of each IMF of Figure $8(\mathrm{~b})$ and the marginal spectrum of radial seismic wave signals in Figure 9, the energy distribution of each frequency band can be obtained. For the convenience of analyzing the energy carried by each frequency band, the whole frequency domain is divided into four frequency bands of $500-800 \mathrm{~Hz}, \quad 300-500 \mathrm{~Hz}, \quad 100-300 \mathrm{~Hz}$, and $0-100 \mathrm{~Hz}$. After squaring the $H(\omega, t)$ in equation (2) and then integrating the frequency, the Hilbert instantaneous energy can be obtained:

$$
\operatorname{IE}(t)=\int_{\omega_{1}}^{\omega_{2}} H^{2}(\omega, t) \mathrm{d} \omega .
$$

According to equation (3), the instantaneous energy proportion of each frequency band of the radial seismic wave signal can be calculated. Similarly, the instantaneous energy proportion of each frequency band of the longitudinal and vertical seismic wave signals in Figure 7 can also be obtained by the same method, and the calculation results are shown in Table 2.

It can be seen from Table 2 that the signal energy of the seismic wave of tunnel expansion blasting is mainly concentrated in the frequency band above $100 \mathrm{~Hz}$. The frequency band of $500-800 \mathrm{~Hz}$ occupies the largest proportion of energy, accounting for $51.560 \%$ of the total energy. The energy of $100-300 \mathrm{~Hz}$ frequency band accounts for $31.016 \%$ of the total energy. The energy proportion of $0-100 \mathrm{~Hz}$ is very small, only accounting for $2.850 \%$ of the total energy, which is consistent with the research results in [25]. 

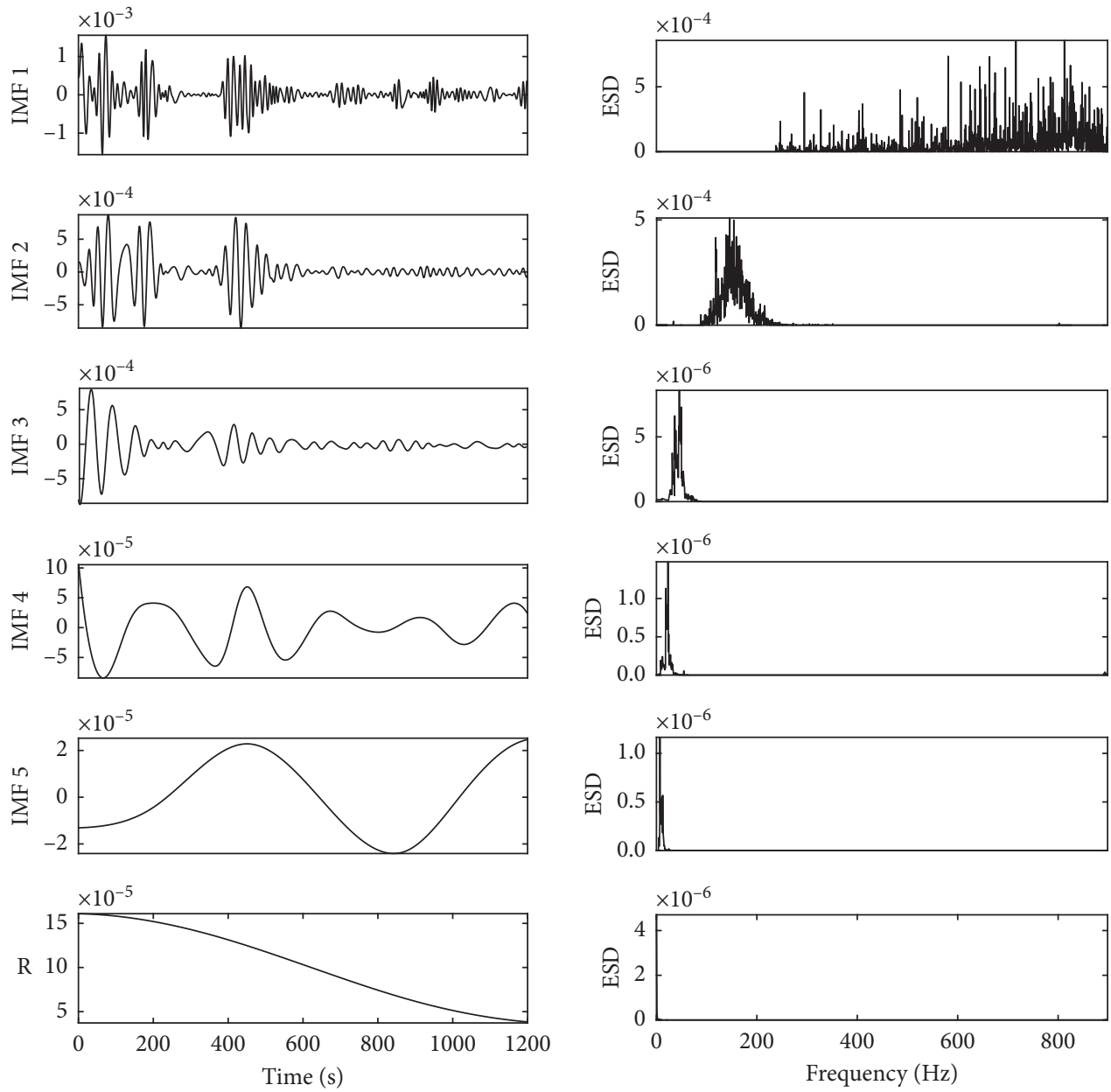

(a)

(b)

FIGURE 8: Results of CEEMD-MPE-HT time-frequency analysis. (a) Decomposition results of EMD. (b) IMF marginal spectrum.

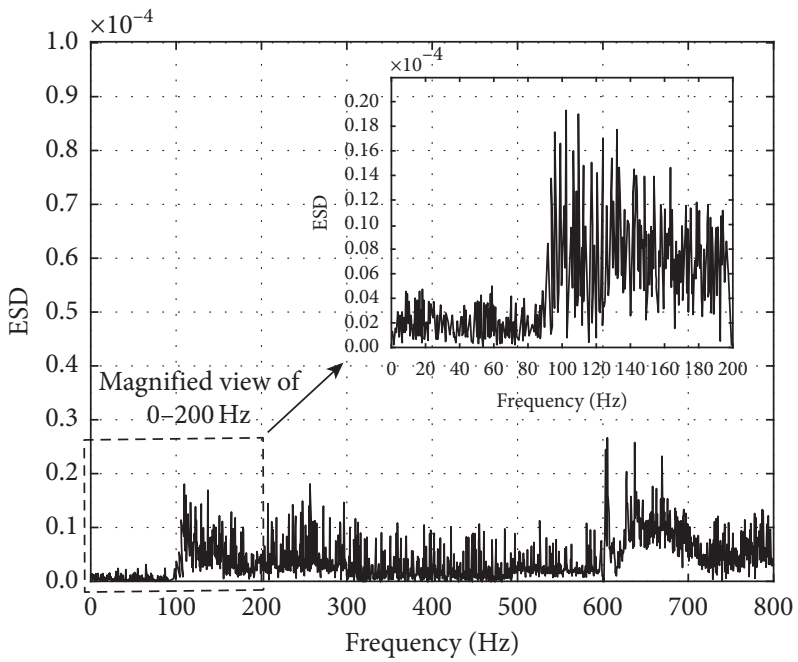

FIgURE 9: Marginal spectrum of the radial seismic wave signal. 
TABLE 2: Energy values of typical seismic wave signals in each frequency band.

\begin{tabular}{|c|c|c|c|c|c|}
\hline \multicolumn{2}{|c|}{ The frequency distribution } & $0-100 \mathrm{~Hz}$ & $100-300 \mathrm{~Hz}$ & $300-500 \mathrm{~Hz}$ & $500-800 \mathrm{~Hz}$ \\
\hline Size of the energy $\left(\mathrm{m}^{2} / \mathrm{s}^{2}\right)$ & $\begin{array}{l}\text { Radial seismic wave signal } \\
\text { Longitudinal seismic wave signal } \\
\text { Vertical seismic wave signal }\end{array}$ & $\begin{array}{l}1.686 \times 10^{-6} \\
1.334 \times 10^{-6} \\
1.027 \times 10^{-6}\end{array}$ & $\begin{array}{l}1.764 \times 10^{-5} \\
1.421 \times 10^{-5} \\
1.243 \times 10^{-5}\end{array}$ & $\begin{array}{l}0.887 \times 10^{-5} \\
0.654 \times 10^{-5} \\
0.542 \times 10^{-5}\end{array}$ & $\begin{array}{l}2.847 \times 10^{-5} \\
2.365 \times 10^{-5} \\
2.149 \times 10^{-5}\end{array}$ \\
\hline $\begin{array}{l}\text { Summary of the e } \\
\text { Energy ratio of each } f\end{array}$ & $\begin{array}{l}\text { y of each frequency band } \\
\text { ency band to the total energy }\end{array}$ & $\begin{array}{l}4.047 \times 10^{-6} \\
2.850 \%\end{array}$ & $\begin{array}{l}4.428 \times 10^{-5} \\
31.016 \%\end{array}$ & $\begin{array}{c}2.083 \times 10^{-5} \\
14.590 \%\end{array}$ & $\begin{array}{l}7.361 \times 10^{-5} \\
51.560 \%\end{array}$ \\
\hline
\end{tabular}

According to [26], the natural frequency of the highway tunnel is about $50 \mathrm{~Hz}$. Therefore, it can be preliminarily judged that the seismic wave signal generated by the expansion blasting of the tunnel on the right side of Loushan will not cause resonance of the existing tunnel on the left side, and the normal operation of the existing tunnel on the left side can be ensured during the blasting construction of the right-side tunnel.

\section{Conclusions}

In tunnel blasting engineering, the hazard effect of blasting vibration can be evaluated by analyzing the time-frequency characteristic parameters contained in seismic wave monitoring signals. To extract the time-frequency information and energy characteristic distribution law contained in blasting signals more truly, the CEEMD-MPE-HT timefrequency analysis model is proposed. Conclusions can be drawn as follows:

(1) CEEMD-MPE algorithm combines the self-adaptability of CEEMD and the ability of MPE to detect the randomness of time series, so that the decomposition results not only retain the original signal characteristic parameters but also suppress the modal confusion phenomenon.

(2) Based on the time-frequency analysis model of the CEEMD-MPE-HT blasting seismic wave signal, the corresponding relationship between frequency and energy contained in the blasting seismic wave signal can be clearly displayed, which is helpful for the identification of blasting vibration signal characteristics.

(3) In the tunnel expansion blasting seismic wave signal, the radial signal has the highest energy, followed by the vertical signal and the longitudinal signal having the lowest energy.

(4) The energy of seismic wave of tunnel expansion blasting is relatively dispersed with frequency distribution, mainly concentrated in a frequency band above $100 \mathrm{~Hz}$, which is much higher than the natural vibration frequency of the adjacent existing tunnel. The energy proportion of $0-100 \mathrm{~Hz}$ is very small, only accounting for $2.850 \%$ of the total energy. The excavation blasting of the tunnel will not cause the resonance of the adjacent existing tunnel.

\section{Data Availability}

All data included in this study are available upon request by contacting the corresponding author.

\section{Conflicts of Interest}

The authors declare that there are no conflicts of interest regarding the publication of this paper.

\section{Acknowledgments}

This work was supported by the Chinese National Natural Science Foundation (Grant no. 41672260).

\section{References}

[1] C.-B. Zhou, N. Jiang, K.-W. Xia, X.-D. Luo, S.-W. Lu, and S.-Y. Hu, Dynamic Action Mechanism and Response Characteristics of Rock Blasting, Science Press, Beijing, China, 2017.

[2] N. Jiang and C. Zhou, "Blasting vibration safety criterion for a tunnel liner structure," Tunnelling and Underground Space Technology, vol. 32, p. 52, 2012.

[3] N. Jiang, C.-B. Zhou, G. Luo, and G.-J. Miao, "Blasting vibration safety criterion of railway tunnel concrete lining," Journal of Central South University (Science and Technology), vol. 43, no. 7, pp. 2746-2750, 2012.

[4] M. Zhao, D. Huang, M. Cao, A. Chi, J. Liu, and Q. Kang, "A energy-based safety evaluation index of blast vibration," Shock and Vibration, vol. 2015, Article ID 698193, 9 pages, 2015.

[5] Y. Peng, Y. Su, L. Wu, and C. Chen, "Study on the attenuation characteristics of seismic wave energy induced by underwater drilling and blasting," Shock and Vibration, vol. 2019, Article ID 4367698, 13 pages, 2019.

[6] H.-X. He, K. Chen, and W.-M. Yan, "Structural seismicdamage assessment based on wavelet packet transformation and time-varying frequencies," Journal of Vibration and Shock, vol. 35, no. 7, pp. 23-30, 2016.

[7] X.-Z. Shi, J.-G. Xue, and S.-R. Chen, "Quadratic time frequency distribution analysis of blasting vibration signal based on bilinear transformation," Journal of Vibration and Shock, vol. 27, no. 12, pp. 131-134, 2008.

[8] X.-B. Li, Y.-P. Zhang, Z.-X. Liu, Y.-J. Zuo, and W.-H. Wang, "Wavelet analysis and Hilbert Huang transform of blasting vibration signal," Explosion and Shock Waves, vol. 25, no. 6, pp. 528-535, 2005.

[9] X.-L. Guan and J.-L. Yan, "The HHT time-frequency power spectrum analysis of the blasting vibration signal," Journal of Vibration and Shock, vol. 32, no. 5, pp. 535-541, 2012.

[10] N.-E. Huang, S. Zheng, S.-R. Luo et al., "The empirical mode decomposition and the Hilbert spectrum for nonlinear and 
non-stationary time series analysis," Proceedings of the Royal Society A, vol. 454, no. 3, pp. 903-995, 1998.

[11] J.-P. Zhao and H. Lin, "Energy distribution and separation of blast wave based on time-frequency energy analysis technology," Chinese Journal of Rock Mechanics and Engineering, vol. 31, no. S1, pp. 3278-3285, 2012.

[12] C.-W. Li, X.-Y. Sun, T.-B. Gao, B.-J. Xie, and X.-M. Xu, "Coal and rock vibration failure and the characteristics of microseismic signals," Journal of China Coal Society, vol. 40, no. 08, pp. 1834-1844, 2015.

[13] T. Wang, M. Zhang, Q. Yu, and H. Zhang, "Comparing the applications of EMD and EEMD on time-frequency analysis of seismic signal," Journal of Applied Geophysics, vol. 83, pp. 29-34, 2012.

[14] J.-R. Yeh and J.-S. Shieh, "Complementary ensemble empirical mode decomposition: a noise enhanced data analysis method," Advances in Adaptive Data Analysis, vol. 2, no. 2, pp. 35-156, 2010.

[15] Z. Wu and N. E. Huang, "Ensemble empirical mode decomposition: a noise-assisted data analysis method," Advances in Adaptive Data Analysis, vol. 01, no. 01, pp. 1-41, 2009.

[16] C. Bandt and B. Pompe, "Permutation entropy: a natural complexity measure for time series," Physical Review Letters, vol. 88, no. 17, pp. 1-5, 2002.

[17] W. Aziz and M. Arif, "Multiscale permutation entropy of physiological time series," in Proceedings of 2005 Pakistan Section Multitopic Conference, Karachi, Pakistan, December 2007.

[18] J.-D. Zheng, T. Liu, R. Meng, and Q.-Y. Liu, "Generalized composite multiscale permutation entropy and PCA based fault diagnosis of rolling bearings," Journal of Vibration and Shock, vol. 37, no. 20, pp. 61-66, 2018.

[19] B.-X. Wang, H.-T. Yang, Q. Wang, X. Gao, and X.-X. Chen, "Bridge vibration signal optimization filtering method based on improved CEEMD-multis-cale permutation entropy analysis," Journal of Jilin University (Engineering and Technology Edition), vol. 50, no. 1, pp. 216-226, 2020.

[20] D.-C. Yao, J.-W. Yang, X.-Q. Cheng, and X. Wang, "Railway rolling bearing fault diagnosis based on muti-scale IMF permutation entropy and SA-SVM classifier," Journal of Mechanical Engineering, vol. 54, no. 09, pp. 168-176, 2018.

[21] J.-D. Zheng, J.-S. Cheng, and Y. Yang, "Modified EEMD algorithm and its applications," Explosion and Shock Waves, vol. 32, no. 21, pp. 21-26, 2013.

[22] J. Li and Q. Li, "Medium term electricity load forecasting based on CEEMDAN-permutation entropy and ESN with leaky integrator neurons," Electric Machines and Control, vol. 19, no. 8, pp. 71-80, 2015.

[23] Y.-P. Zhang and X.-B. Li, "Application of Hilbert-Huang transform in blasting vibration signal analysis," Journal of South China University of Technology (Natural Science Edition), vol. 5, pp. 168-173, 2005.

[24] X. Zheng, Z.-Y. Hao, Z.-G. Lu, and J. Yang, "Separation of piston-slap and combustion shock excitations via MEEMD method," Journal of Vibration and Shock, vol. 31, no. 06, pp. 109-113, 2012.

[25] X.-G. Yang, D.-Q. Shu, W.-B. Lu, and X.-J. Gao, "Study on energy distribution characteristics of seismic waves induced by different forms of blasting resource," Journal of Sichuan
University(Engineering Science Edition), vol. 42, no. 1, pp. 30-34, 2010.

[26] Y. Fan, F. Wang, W.-B. Lu et al., "Safety evaluation of deep tunnel blasting excavation vibration considering low-frequency amplification effect of transient unloading of in-situ stress," Chinese Journal of Rock Mechanics and Engineering, vol. 37, no. s2, pp. 4184-4197, 2018. 